\title{
Synaptic and circuit development of the primary sensory cortex
}

\author{
Se-Young Choi (i)
}

\begin{abstract}
Animals, including humans, optimize their primary sensory cortex through the use of input signals, which allow them to adapt to the external environment and survive. The time window at the beginning of life in which external input signals are connected sensitively and strongly to neural circuit optimization is called the critical period. The critical period has attracted the attention of many neuroscientists due to the rapid activity-/experience-dependent circuit development that occurs, which is clearly differentiated from other developmental time periods and brain areas. This process involves various types of GABAergic inhibitory neurons, the extracellular matrix, neuromodulators, transcription factors, and neurodevelopmental factors. In this review, I discuss recent progress regarding the biological nature of the critical period that contribute to a better understanding of brain development.
\end{abstract}

\section{Biological nature of the critical period}

Many vertebrate animals, including humans, recognize their environment and learn how to live using experiences from the early time window of their lives. During this time, the nervous system actively develops neural circuits in accordance with experience inputs. The phenomenon of brain function is at first immature and is then optimized according to experience, as can be observed in many animals. So, how does this experience change the brain? This has long attracted the attention of many scientists.

\section{Early studies of the critical period}

This so-called "experience" from the external environment is accommodated by the cerebral cortex. In order for this experience to be properly accepted as an input signal, a neural circuit that is finely optimized by the input signal is needed. This optimization requires both fine tuning of the synapse structure and synaptic plasticity. It should be noted that the receiving of input signals, which are important for the fine tuning of synapses during this

\footnotetext{
Correspondence: S-Y. Choi (sychoi@snu.ac.kr)

'Department of Physiology and Dental Research Institute, Seoul National

University School of Dentistry, Seoul 03080, Republic of Korea
}

time, is not an experience that occurs at any time but rather is an experience that occurs at an early "specific time" in life. The critical period is when sensory input is strongly connected to the optimization of the neural circuit. Indeed, the ability to control synaptic function for certain types of learning and memory is not constant over a lifetime, often reaching a peak at a specific time after birth and generally decreasing at various rates as age increases. A representative example of this learning is the parental imprinting of graylag geese by Austrian zoologist and founder of modern ethology Lorenz in 1935, which allows a subject to be considered as a mother during a short period of time after the goslings hatch (13-16 h after birth).

A more detailed examination of this phenomenon was done in a study of the cat visual cortex. The binocular region of the vertebral animal's visual cortex receives signals from both eyes, but neurons in certain regions of the visual cortex are more likely to receive and process input signals from one eye. This is called ocular dominance ${ }^{1}$. Studies of ocular dominance in cats and monkeys have shown that several cells that perceive similar visual characteristics are located together in a single column structure and that these structures receiving the input from each eye act in a competitive manner ${ }^{2}$. It is 
important to note that these changes make it possible for a particular period of experience to permanently change neural circuits on a large scale ${ }^{3}$. The trans-neuronal transport technique, which stains neurons involved in the eye or lateral geniculate nucleus, provides anatomical evidence for the experience-dependent changes that occur in ocular dominance and for the presence of a critical period. Interestingly, when one eye is impaired (i.e., "monocular deprived"), it changes the ocular dominance in the visual cortex; this effect is maximized during the first few weeks of life (4-8 weeks for cats) ${ }^{4}$. Mice also have a critical period in the visual cortex, which occurs during days $21-32$ of age $^{5,6}$. In addition to the visual cortex, early studies investigating critical periods have been performed in the somatosensory cortex. When one of the fingers was cut, the area within the somatosensory cortex changed toward receiving information from the adjacent fingers ${ }^{7}$. If a specific whisker of a rat or mouse is removed, then the sensory cortex that receives the signal from the whisker will lose synaptic connections.

\section{Early studies of the mechanism of critical period}

Since the existence of a critical period was discovered $>40$ years ago $^{4}$, subsequent researches have sought to identify the biological mechanism of the critical period. One of the most intensively studied is GABAergic inhibitory neurons. Even before the molecular nature of the critical period was studied, GABAergic neurons had been considered to be critical for the synaptic plasticity of the cerebral cortex. In the cerebral cortex of a young animal which is not within the critical period, long-term potentiation (LTP) can be induced successfully in layer $2 / 3$, whereas LTP does not occur in adult animals ${ }^{8-10}$. Other evidence suggests that the inhibitory circuit of layer 4 may be involved in altering synaptic properties during the critical period. In other words, during this period inhibition is relatively weak, the door of change is open, and the synaptic properties can be changed. Susumu Tonegawa's research group developed a transgenic mouse line whose forebrain specifically overexpresses brain-derived neurotrophic factor (BDNF) under the control of the $\alpha-\mathrm{Ca}^{2+} /$ calmodulin-dependent protein kinase II $(\alpha \mathrm{CaM}-$ KII) promoter ${ }^{11}$. Interestingly, the BDNF-overexpressing transgenic mice shows earlier development of GABAergic inhibitory neurons as well as earlier start of critical period. The activity of inhibitory neurons was measured in darkreared mice by harvesting these neurons in a dark room immediately after birth; their inhibitory activity was observed to be significantly lower in comparison with normal mice. In addition, the inhibition was restored to almost normal levels when the dark-reared mice were exposed to light for 2 days right before the time when the critical period was almost finished ${ }^{12}$. In addition, it has been reported that the ocular dominance shift that normally occurs during the critical period was impaired in transgenic animals with decreased GABA synthesis ${ }^{13,14}$. In these animals, long-term administration of benzodiazepine, a $\mathrm{GABA}_{\mathrm{A}}$ receptor agonist, restored long-term depression (LTD) induction ${ }^{14}$ and ocular dominance plasticity $^{13,15}$. Thus the development of BDNF-induced inhibitory neurons can be hypothesized to lead to synaptic plasticity, allowing changes to occur during the critical period. Recently, Lamberto Maffei's research group has further reported that normal inhibition and ocular dominance plasticity occur in the BDNF-overexpressing transgenic mice, even in dark-rearing conditions ${ }^{16}$.

\section{Functional key player: GABA and GABAergic neurons}

Based on this research, many follow-up studies have characterized the GABAergic neurons involved in the critical period. GABAergic inhibitory interneurons in the cerebral cortex are classified according to their unique biomarkers, including parvalbumin (PV), somatostatin (SOM), vasoactive intestinal peptide (VIP), and ionotropic serotonin receptor 5HT3a $(5 \mathrm{HT} 3 \mathrm{aR})^{128}$. Among these, inhibitory neurons modulating the critical period are $\mathrm{PV}(+)$ inhibitory neurons, also called fastspiking interneurons ${ }^{17}$. PV expression in these interneurons is considered to be consistent during the critical period $^{18}$. PV $(+)$ neurons mainly act on the soma and proximal dendrites of excitatory neurons, inducing perisomatic inhibition, thereby regulating the firing and backpropagation of action potentials in excitatory neurons ${ }^{19-21}$. The development of PV $(+)$ neurons is induced by BDNF signaling in an experience-dependent manner ${ }^{11,22}$. During the critical period, GABAergic inhibition is increased in cortical layers $2-3$, and glutamatergic signaling is weakened to decrease the excitatory-inhibitory balance ${ }^{23}$. The function of PV $(+)$ cells, as well as the role of other inhibitory neurons, has also been extensively studied at the circuit level. For example, layer 5b SOM (+) neurons regulate layer 4 spiny stellates neurons, thus controlling early development in the thalamocortical recipient layer ${ }^{24}$. The importance of the disinhibitory circuit of VIP-spiny stellate neurons is also attracting attention ${ }^{25}$. During the critical period, excitatory circuits were found to be selectively changed only in the principal cells, PV $(+)$ and SOM $(+)$ cells, whereas inhibitory and thalamocortical synapses did not change ${ }^{26}$. The ionotropic $\mathrm{GABA}_{\mathrm{A}}$ receptor plays an important role during the critical period in mediating GABA signaling. The GABA receptor alpha 1 subunit, which allows fast-spiking activity, has been reported to play an important role during the critical period ${ }^{27}$. In addition, the conductivity of the $\mathrm{GABA}_{\mathrm{A}}$ channel itself can be altered by BDNF signaling during the critical period ${ }^{28}$. Another characteristic of $\mathrm{GABA}_{\mathrm{A}}$ receptors is that they exhibit an excitatory function at the 


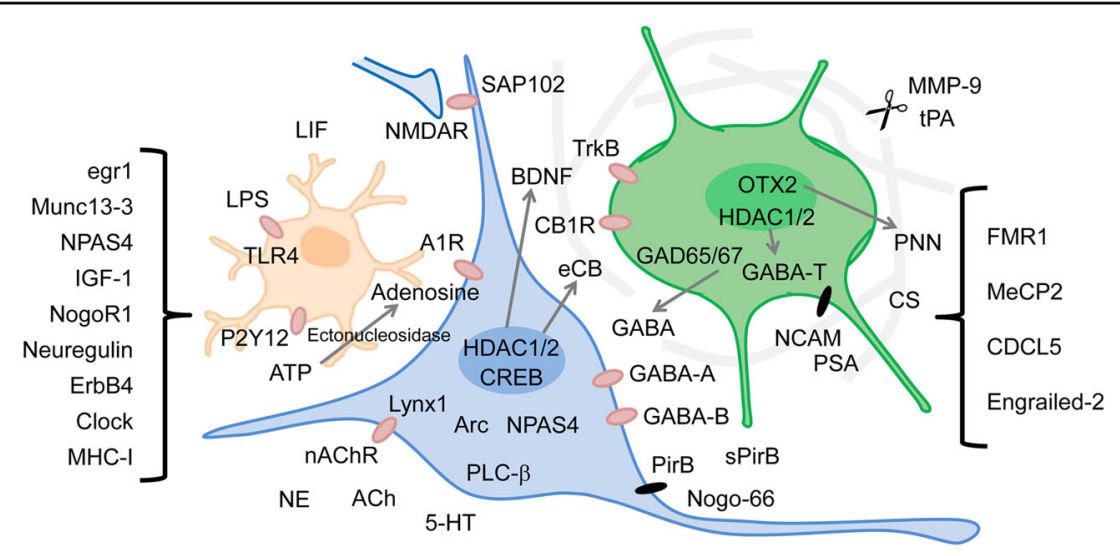

Fig. 1 Factors modulating the critical period. Factors that are known to control the critical period to date are depicted in the area where they operate predominantly. Factors with unclear action sites are listed on the left side and the neurodevelopmental disease-related factors are listed on the right side. The green cell is a PV (+) inhibitory neuron, the blue cell is a pyramidal neuron, and the orange cell is a glia cell (microglia).

Perineuronal net is depicted in pale gray. Receptors or channels are red and cell adhesion molecules are black

onset of development and then convert to exerting an inhibitory effect. This is due to the altered expression of the KCC2 transporters ${ }^{29,30}$. Interestingly, brief termination of this depolarizing GABA signaling decreases inhibitory neuronal transmission and the expression of $\mathrm{BDNF}$ and perineuronal net (PNN) components ${ }^{31}$. Finally, the synthesis and reabsorption of GABA is also attracting attention as a regulator of the critical period ${ }^{32}$. So, how does this GABAergic signaling regulate the entire neuronal circuit with which the excitatory nerves are associated? First, the inhibitory neural circuit affects Hebbian synaptic plasticity, such as LTP and LTD. This synaptic plasticity plays an important role in maturing the circuit in the primary sensory cortex during the critical period ${ }^{33,34}$. Another effect of inhibitory neuronal circuit is spike timing-dependent plasticity (STDP). STDP is a type of plasticity formed by the timing of two action potentials firing in the presynaptic cell and postsynaptic cell, and GABAergic signaling modulates the action potential backpropagation and perisomatic inhibition $^{35,36}$.

\section{Structural key player: perineuronal net and extracellular matrix (ECM)}

The other feature to be considered with PV $(+)$ neurons is $\mathrm{PNN}^{37}$. The frontiers in critical period research indicate that the structural factors controlling spine formation, such as the ECM, may be important because the dendritic spine motility decreases at the end of the critical period ${ }^{38}$. In actuality, the ECM exists outside of neurons to physically support cells and optimize neurotransmission. The PNN is one of the typical ECM components composed of brevican, aggrecan, neurocan, phophacan, and tenascin$\mathrm{R}^{39}$. Dr. Maffei's group removed the ECM by destroying the extracellular glycoproteins and found that ocular dominance plasticity was re-introduced in the older visual cortex ${ }^{40}$. Additionally, removal of the ECM promotes dendrite synthesis ${ }^{41}$. It is well known that neuronal ECM is important for structural fine-tuning of axons and synapses $^{42}$. In particular, PNNs are distributed around PV $(+)$ neurons and regulates synaptic input to these neurons. If visual or somatosensory experiences are blocked during the critical period, the development of PNNs decreases and plasticity is maintained ${ }^{43}$. In addition, since chondroitin sulfate (a major component of PNNs) accumulates in PNNs during the critical period, the beginning of ocular dominance plasticity disappears in CSGalNAcT1 knockout (KO) mice, which lack chondroitin sulfate synthesis ${ }^{44}$. Removal of PNNs also changes the excitatory-inhibitory balance by lowering the inhibitory activity of the neuronal network into a juvenile state ${ }^{45}$. In addition, Ngr1 (a receptor of chondroitin sulfate) terminates the critical period by inhibiting the monocular deprivation-mediated reduction of intracortical input to the PV $(+)$ neuron-connected disinhibitory microcircuit $^{46}$. Cell adhesion-related molecules that regulate these extracellular environments with PNNs and synaptogenesis are also emerging as other modulators. Typical representatives are neural cell adhesion molecules (NCAMs) and polysialic acid (PSA ${ }^{47}$. NCAM and PSA regulate ocular dominance plasticity by modulating the maturation of the GABAergic circuits ${ }^{48}$. There have been reports that the protein degradation activity of tissue plasminogen activator, which degrades the ECM, is required for the effects of monocular deprivation ${ }^{49,50}$. Cartilage link protein also regulates the formation of PNNs associated with the critical period ${ }^{51}$. Recently, it has also been found that light reintroduction causes matrix 
metalloproteinase-9-mediated ECM degradation and critical period reactivation ${ }^{52}$.

\section{Critical period controllers}

What, then, can control the critical period? In the early studies investigating critical period modulators, there were early attempts to detect genes that were differentially expressed before and after the critical period. CREB $^{53}$, EGR1 ${ }^{54}$, EGR1/ZIF268 ${ }^{55}$, Munc13- $3^{56}$, and major histocompatibility complex (MHC)- $\mathrm{I}^{57}$ were found to be candidates for critical period modulators. These attempts have been extended by a series of recent advancements in molecular biology techniques (Fig. 1). Recently, the transcriptome of OTX2, a key critical period-related transcription factor (described below), was used to identify Kv3.1 and OXY1 by using interneuron-specific RNA-seq/ Chip-seq techniques ${ }^{58}$.

\section{Neuromodulators}

One of the first factors to control the critical period is the neuromodulator. Earlier studies have revealed that the functions and circuit optimization of the primary sensory cortex are modulated by cholinergic ${ }^{59}$, noradrenergic ${ }^{59,60}$, and serotonergic ${ }^{61}$ inputs from extra-retinal pathways. A following study showed that the G-protein coupled receptor-mediated phospholipase C (PLC) signaling pathway modulates $\mathrm{N}$-methyl-D-aspartate receptor-dependent LTD induction in the visual cortex ${ }^{62,63}$. These studies have been conducted steadily to date, and chronic treatment with fluoxetine, an selective serotonin reuptake inhibitor antidepressant, has been shown to reintroduce ocular dominance plasticity in adults ${ }^{64,65}$, suggesting that 5 - HT may control the plasticity of the critical period. It is also known that the expression of LYNX1, which inhibits nicotinic signaling, controls the critical period by controlling cholinergic innervation and the excitatory-inhibitory balance ${ }^{66}$. Recently, the GABA-B receptor has also been shown to modulate ocular dominance plasticity ${ }^{67}$. Also, the synthesis of adenosine by ectonucleotidases and the disruption of the adenosine A1 receptor have been reported to be important for juvenile plasticity in the auditory cortex ${ }^{68}$. In particular, endocannabinoids induce GABAergic maturation of fastspiking PV $(+)$ neurons by regulating inhibitory LTD $^{69}$. Treatment with a cannabinoid type 1 (CB1) receptor agonist in the dark-exposed state of adult rats results in the maturation of inhibition ${ }^{70}$. BDNF results in the secretion endocannabinoids through TrkB signaling in the visual $\operatorname{cortex}^{70,71}$. The BDNF-endocannaboid axis is also regulated by other signals associated with G-protein coupled receptors and PLC-beta signaling, such as $\mathrm{mGluR}^{72,73}$.

\section{Transcriptional factors and immediate early genes}

To understand how inhibitory circuits are reconstituted by input signals given in the early days of life, attention should be paid to the activity dependency of inhibitory circuit development. I have already discussed activitydependent BDNF action in depth ${ }^{11,74,75}$. The main hypothesis is that pyramidal neurons secrete BDNF and that the inhibitory neurons develop as a result of this secreted $\mathrm{BDNF}^{76,77}$. However, the upstream signaling that causes the pyramidal neurons to secrete BDNF in an activity-dependent manner is still unclear. Over the past several years, attempts have been made to understand this in terms of activity-dependent transcriptional modulation. Among them, there is a transcription factor called orthodenticle homeobox 2 (OTX2). OTX2 defects causing eye malformation in humans distort eye structure and function to various degrees, depending on the expression levels of the gene ${ }^{78}$. Interestingly, OTX2 is produced in the retina but is secreted and transported through the visual ascending pathway, where it is then absorbed into the cell after binding to PNNs on the surface of PV (+) neurons in the visual cortex ${ }^{129}$. If OTX2 and PNN are blocked by manipulating the glycosaminoglycan-binding sequence of OTX2, reactivity of ocular dominance plasticity occurs ${ }^{79}$. OTX2 regulates a variety of critical periodrelated factors, including IGF ${ }^{80}$. Neuronal Per Arnt Sim domain protein 4 (NPAS4) is another neuronal activitydependent transcription factor ${ }^{81}$ whose expression is well known to modulate neurite outgrowth and the function of synaptic proteins ${ }^{82}$. Interestingly, NPAS4 regulates experience-dependent GABAergic synapse development by expressing factors necessary for the formation and maintenance of inhibitory synapses innervating excitatory neurons ${ }^{83}$.

$A r c$ is an activity-dependent immediate early gene in the neural system, and its importance has long been understood. Increased Arc expression in adult mice results in restoration of ocular dominance plasticity, such as juvenile-like plasticity and LTD induction in the adult visual cortex, and impaired LTD in Arc $\mathrm{KO}$ mice was restored by a protein synthesis inhibitor ${ }^{84}$. Arc mRNA expression itself was also increased in the critical period, and this increase was found to be important for juvenilelike plasticity. Another well-known factor is CREB. Visual stimulation in juvenile mice has been shown to increase CREB-induced transcription, and this effect has been shown to decrease in adults ${ }^{85}$. In addition, visual experience during the critical period has been reported to increase the expression of miR-132, a CREB-induced microRNA ${ }^{86,87}$. Interestingly, these mechanisms involve epigenetic modulation by posttranslational modification of histone proteins ${ }^{43}$. This idea is based on the finding that histone deacetylase (HDAC) inhibitors regulate the critical period in the visual cortex ${ }^{85}$ and that HDAC1 ${ }^{88}$ and HDAC2 ${ }^{89}$ affect the functions of PV $(+)$ neurons in the critical period. 
Glia

The last issue to consider is the functions of glial cells during the critical period. It has been reported that central nervous system myelination is associated with the termination of the critical period ${ }^{90}$ and that the injection of immature astrocytes can reintroduce ocular dominance plasticity in older cats ${ }^{91}$. These reports have expanded the interest in how glia regulates the critical period. In recent decades, accumulating evidence has shown that glia is not simply a backbone for neurons but actively communicates with neurons to regulate brain function ${ }^{92-94}$. For example, astrocytes can communicate with neurons and develop inhibitory circuits via their $\mathrm{CB} 1$ receptors ${ }^{95}$. Recently, it has been found that ocular dominance plasticity disappears when the P2Y12 receptor, which selectively exists in non-activated microglia and causes early injury responses, is eliminated ${ }^{96}$. In addition, a systemic injection of lipopolysaccharides during the critical period has shown that inflammation affects the critical period ${ }^{97}$. Recently, studies on glial factors have been carried out. It is reported that infusion of leukemia inhibitory factor affects MT-4-mediated expression of PV, Kv3.1, and GAD-65 and inhibits neuronal development during the critical period ${ }^{98}$. In the future, investigation into glial regulation of the critical period is expected to highlight many interesting issues.

\section{Others}

So, how did the search and identification of the factors related to the critical period come about? As an early study, it was found that the Cpg (candidate plasticity gene) family is expressed when the dentate gyrus of the hippocampus is treated with kainate to induce strong firing. It is known that CPG15 is expressed at the beginning of the critical period ${ }^{99}$. Many critical periodrelated molecules have since been discovered. Many developmental modulators, such as IGF-1 ${ }^{100}$, Nogo-66 receptor ${ }^{101}$, NogoR $1^{102}$, neuregulin-1 and ErbB4 signaling ${ }^{103,104}$, and neurogranin ${ }^{105}$, have been shown to control the critical period. One of the interesting factors is the Clock gene, which is related to the circadian rhythm. Clock KO animals showed PV $(+)$ neuron development and delay of ocular dominance plasticity $^{106}$. This results in the new question of whether other factors that regulate circadian rhythms, such as BMAL, are also involved in the critical period. Another such factor is Paired-immunoglobulin-like receptor B (PirB), which was originally known to regulate axonal regeneration by binding to $\mathrm{MHC}$ class I and Nogo ${ }^{107}$. Interestingly, PirB controls ocular dominance plasticity $^{108}$. Acute interruption of PirB expression in the adult leads to new synapse formation, resulting in increased L5 miniature excitatory synaptic current frequency, increased spine density, and restoration of amblyopia ${ }^{109}$. Since PirB is also expressed in microglia, the role of microglia in the critical period control function of PirB may be worthy of investigation.

\section{Neurodevelopment and critical period Neurodevelopmental diseases}

Developmental changes in the critical period can often overlap with the pathology of certain neurodevelopmental diseases. Therefore, if there are interesting molecules related to the developmental diseases, it could be important to check the importance of these factors in association with critical period. In FMR1 KO mice, there is delayed maturation of fast-spiking GABAergic neurons in the sensory cortex due to BDNF-TrkB signal distortion and cortical developmental abnormalities during the critical period ${ }^{110}$. PV $(+)$ neuron-specific MeCP2 conditional $\mathrm{KO}$ mice lack experience-dependent plasticity during the critical period, whereas SOM $(+)$ neuronspecific and glutamatergic neuron-specific MeCP2 $\mathrm{cKO}$ mice show no such effect in the critical period ${ }^{111}$. In a mouse model involving a factor associated with Rett syndrome (CDKL5 -/y mice), PNN expression levels in the V1 area are lower but the number of PV $(+)$ neurons is increased, resulting in more innervation to pyramidal neurons and a change in the excitatory-inhibitory balance $^{112}$. SYNGAP1 haploinsufficiency causing neurodevelopmental disease affects dendrite growth and spine plasticity in the neocortex ${ }^{113}$. In addition, the altered critical period can be observed in animal models of autism spectrum disorder. Semaphorin 7A, a factor of autism spectrum disorder $15 \mathrm{q} 24$ microdeletion syndrome, is important for feed-forward GABAergic inhibition in the somatosensory barrel cortex and is also important for barrel formation and layer 4 circuit development ${ }^{114}$. Autism spectrum disease-associated Engrailed-2 KO animals show an increase in PV $(+)$, SOM $(+)$, and NPY $(+)$ neurons at postnatal day 30 and a decrease in SOM $(+)$ and NPY $(+)$ neurons in adults. The visual function of Engrailed-2 $\mathrm{KO}$ animals is normal, but their binocularity is increased, and there is no response to brief monocular deprivation during the critical period ${ }^{115}$. In addition, SAP102 KO animals, a model of intellectual disability, show normal barrel formation with a reduced number of thalamocortical axons, as well as altered kinetics of the NMDA receptor ${ }^{116}$.

\section{Changes in development of other cortex}

Then how can we distinguish between critical period changes and neurodevelopmental changes? To date, this is not clear. One simple issue to be considered is whether these factors exert these specified effects only in the primary sensory cortex or also elsewhere in the brain. Furthermore, it is necessary to examine whether other cortex regions show changes in circuit formation during the 
critical period. For example, a phenomenon similar to the critical period has been found in some other cortices, such as the medial prefrontal cortex (mPFC). Neurons in layer 5 of the mPFC can be classified as $\mathrm{PH}$ cells or non- $\mathrm{PH}$ cells, according to the existence of $\mathrm{H}$-current. After 2 weeks of weaning, social isolation decreases the action potential firing rate and synaptic input to $\mathrm{PH}$ cells in mice ${ }^{117}$. In addition, when NMDA is blocked with MK-801, development of the PV $(+)$ and $\mathrm{CB}(+)$ neurons of the mPFC is decreased ${ }^{118}$. It is reported that fluoxetine treatment increases PCA-NCAM and GAD95/97 expression, decreases the number of PV $(+)$ neurons covered with PNNs, and changes the interneuron structure of the $\mathrm{mPFC}^{119}$. Therefore, the extent to which these developmental modulators are involved in experience-dependent circuit development within the critical period is very diverse and unclear. It is also unclear how many of the neuronal and cognitive defects seen in a $\mathrm{KO}$ animal model of a certain factor are related to the critical period. However, if these relationships are clarified in the future, it may be possible to derive a method for restoring normal structure and function in these developmental disorders based on critical period reinstate approaches.

\section{Changes in adult cortex}

Since the concept of the critical period was first associated with the earlier time window of life, the idea that plasticity disappears in adults has prevailed for a while. However, it has been shown (mostly in rodents) that ocular dominancy plasticity can be controlled even in adult age ${ }^{120}$. Taken together, the ocular dominancy plasticity mechanism in juveniles and adults appears to be somewhat mixed. However, the detailed mechanisms for reintroducing ocular dominancy plasticity in adults might distinguish the "critical period" from "neurodevelopment". One interesting finding is that experience from an enriched environment modulates adult plasticity ${ }^{121,122}$. Studies of other critical period regulators are underway to reveal these mechanisms. One such study showed that an enriched environment increases histone $\mathrm{H} 3$ acetylation when reopening the critical period ${ }^{123}$. Another reason for the significance of adult plasticity is that it may contribute to the development of treatments for human sensory impairments, such as amblyopia ${ }^{124}$. A series of treatments, including transcranial direct current stimulation ${ }^{125}$ and high-frequency transcranial electrical stimulation ${ }^{126}$, have been tried to cure amblyopia. Studies of the critical period study are helpful in determining the optimal timing of those treatments. In fact, transcranial magnetic stimulation causes a decrease in PV $(+)$ neurons, which is not effective before postnatal day 30 but has been shown to be effective after $^{127}$.

\section{Conclusions}

Finally, why are neuroscientists interested in the critical period? In other words, what is the academic appeal of the critical period? First, the rapid changes of the critical period during brain development are intriguing. In adults, it is necessary to perform brain functions (such as sensation, decision-making, and movement) stably based on established neural circuits, so the plasticity of circuits is not necessarily active. The critical period is a time window where such changes are very active, and there are many interesting and significant changes in cerebral cortex. Another reason for this interest may arise from the critical period's activity- and/or experiencedependent mechanisms. Activity dependency is a very important topic in many areas of neuroscience. Since the critical period is basically the time when neuronal circuits are optimized by external experience, the experience input triggers all changes. This feature is different from the normal neurodevelopment process that develops naturally regardless of external stimuli. The circuit-behavior correlation is another factor that makes critical period interesting. The sensory experience can be manipulated easily and precisely by controlling the input (shining a light, touching a whisker, etc.) or temporarily controlling the sensory organs (temporary operation of the eyelid, shaving a certain whisker, etc.). In particular, since these changes occur in the sensory cortex, it is also beneficial to apply recently developed imaging technics (e.g., in vivo two-photon imaging). Lastly, these findings can be developed into human therapeutic applications, such as amblyopia treatment and cochlear implants. The technique of treating sensory defects based on critical period studies will open up new horizons in rehabilitation medicine. The features of these various critical period studies will be attractive to many neuroscientists in the future.

\section{Acknowledgements \\ I thank Mr. Heeyoon Lee for preparing manuscript with references. This work was supported by the National Research Foundation of Korea} (2016R1A2B4006811).

Conflict of interest

The authors declare that they have no conflict of interest.

\section{Publisher's note}

Springer Nature remains neutral with regard to jurisdictional claims in published maps and institutional affiliations

Received: 21 November 2017 Accepted: 6 December 2017.

Published online: 9 April 2018

\footnotetext{
References

1. Hubel, D. H. \& Wiesel, T. N. Receptive fields, binocular interaction and functional architecture in the cat's visual cortex. J. Physiol. 160, 106-154 (1962).
} 
2. Hubel, D. H. \& Wiesel, T. N. Stereoscopic vision in macaque monkey. Cells sensitive to binocular depth in area 18 of the macaque monkey cortex. Nature 225, 41-42 (1970).

3. Hubel, D. H. \& Wiesel, T. N. The period of susceptibility to the physiological effects of unilateral eye closure in kittens. J. Physiol. 206, 419-436 (1970).

4. Hubel, D. H. \& Wiesel, T. N. Shape and arrangement of columns in cat's striate cortex. J. Physiol. 165, 559-568 (1963).

5. Gordon, J. A. \& Stryker, M. P. Experience-dependent plasticity of binocular responses in the primary visual cortex of the mouse. J. Neurosci. 16 3274-3286 (1996).

6. Drager, U. C. Observations on monocular deprivation in mice. J. Neurophysiol. 41, 28-42 (1978)

7. Merzenich, M. M. et al. Somatosensory cortical map changes following digit amputation in adult monkeys. J. Comp. Neurol. 224, 591-605 (1984).

8. Kirkwood, A., Lee, H. K. \& Bear, M. F. Co-regulation of long-term potentiation and experience-dependent synaptic plasticity in visual cortex by age and experience. Nature 375, 328-331 (1995).

9. Kirkwood, A., Rioult, M. C. \& Bear, M. F. Experience-dependent modification of synaptic plasticity in visual cortex. Nature 381, 526-528 (1996)

10. Kirkwood, A. \& Bear, M. F. Hebbian synapses in visual cortex. J. Neurosci. 14, 1634-1645 (1994).

11. Huang, Z. J. et al. BDNF regulates the maturation of inhibition and the critical period of plasticity in mouse visual cortex. Cell 98, 739-755 (1999).

12. Morales, B., Choi, S. Y. \& Kirkwood, A. Dark rearing alters the development of GABAergic transmission in visual cortex. J. Neurosci. 22, 8084-8090 (2002)

13. Fagiolini, M. \& Hensch, T. K. Inhibitory threshold for critical-period activation in primary visual cortex. Nature 404, 183-186 (2000).

14. Choi, S. Y., Morales, B., Lee, H. K. \& Kirkwood, A. Absence of long-term depression in the visual cortex of glutamic acid decarboxylase-65 knock-out mice. J. Neurosci. 22, 5271-5276 (2002).

15. Hensch, T. K. et al. Local GABA circuit control of experience-dependent plasticity in developing visual cortex. Science 282, 1504-1508 (1998).

16. Gianfranceschi, L. et al. Visual cortex is rescued from the effects of dark rearing by overexpression of BDNF. Proc. Natl. Acad. Sci. USA 100 12486-12491 (2003).

17. Hensch, T. K. Critical period plasticity in local cortical circuits. Nat. Rev. Neurosci. 6, 877-888 (2005)

18. del Rio, J. A., de Lecea, L., Ferrer, I. \& Soriano, E. The development of parvalbumin-immunoreactivity in the neocortex of the mouse. Brain Res. Dev. Brain Res. 81, 247-259 (1994)

19. Tsubokawa, H. \& Ross, W. N. IPSPs modulate spike backpropagation and associated $\left[\mathrm{Ca}^{2+}\right]_{\mathrm{i}}$ changes in the dendrites of hippocampal CA1 pyramidal neurons. J. Neurophysiol. 76, 2896-2906 (1996).

20. Sohal, V. S., Zhang, F., Yizhar, O. \& Deisseroth, K. Parvalbumin neurons and gamma rhythms enhance cortical circuit performance. Nature 459, 698-702 (2009).

21. Gulyas, A. I. \& Freund, T. T. Generation of physiological and pathologica high frequency oscillations: the role of perisomatic inhibition in sharpwave ripple and interictal spike generation. Curr. Opin. Neurobiol. 31, 26-32 (2015).

22. Mizuno, K., Carnahan, J. \& Nawa, H. Brain-derived neurotrophic factor promotes differentiation of striatal GABAergic neurons. Dev. Biol. 165, 243-256 (1994).

23. Kannan, M., Gross, G. G., Arnold, D. B. \& Higley, M. J. Visual deprivation during the critical period enhances layer 2/3 GABAergic inhibition in mouse V1. J. Neurosci. 36, 5914-5919 (2016).

24. Marques-Smith, A et al. A transient translaminar GABAergic interneuron circuit connects thalamocortical recipient layers in neonatal somatosensory cortex. Neuron 89, 536-549 (2016).

25. Fu, Y., Kaneko, M., Tang, Y., Alvarez-Buylla, A. \& Stryker, M. P. A cortical disinhibitory circuit for enhancing adult plasticity. Elife 4, e05558 (2015)

26. Miao, Q. et al. Selective maturation of temporal dynamics of intracortical excitatory transmission at the critical period onset. Cell Rep. 16, 1677-1689 (2016)

27. Fagiolini, M. et al. Specific GABAA circuits for visual cortical plasticity. Science 303, 1681-1683 (2004)

28. Gao, M. et al. Rebound potentiation of inhibition in juvenile visual cortex requires vision-induced BDNF expression. J. Neurosci. 34, 10770-10779 (2014)

29. Wang, D. D. \& Kriegstein, A. R. Defining the role of GABA in cortical development. J. Physiol. 587, 1873-1879 (2009).
30. Ben-Ari, Y. The GABA excitatory/inhibitory developmental sequence: a personal journey. Neuroscience 279, 187-219 (2014).

31. Deidda, G. et al. Early depolarizing GABA controls critical-period plasticity in the rat visual cortex. Nat. Neurosci. 18, 87-96 (2015).

32. $\mathrm{Wu}, \mathrm{X}$. et al. GABA signaling promotes synapse elimination and axon pruning in developing cortical inhibitory interneurons. J. Neurosci. 32, 331-343 (2012).

33. Smith, G. B., Heynen, A. J. \& Bear, M. F. Bidirectional synaptic mechanisms of ocular dominance plasticity in visual cortex. Philos. Trans. R. Soc. Lond. B. Biol. Sci. 364, 357-367 (2009).

34. Feldman, D. E. Synaptic mechanisms for plasticity in neocortex. Annu. Rev. Neurosci. 32, 33-55 (2009).

35. Lisman, J. \& Spruston, N. Postsynaptic depolarization requirements for LTP and LTD: a critique of spike timing-dependent plasticity. Nat. Neurosci. 8 839-841 (2005).

36. Caporale, N. \& Dan, Y. Spike timing-dependent plasticity: a Hebbian learning rule. Annu. Rev. Neurosci. 31, 25-46 (2008).

37. Kadomatsu, K. \& Sakamoto, K. Sulfated glycans in network rewiring and plasticity after neuronal injuries. Neurosci. Res. 78, 50-54 (2014).

38. Grutzendler, J., Kasthuri, N. \& Gan, W. B. Long-term dendritic spine stability in the adult cortex. Nature 420, 812-816 (2002).

39. Ueno, H., Suemitsu, S., Okamoto, M., Matsumoto, Y. \& Ishihara, T. Sensory experience-dependent formation of perineuronal nets and expression of Cat-315 immunoreactive components in the mouse somatosensory cortex. Neuroscience 355, 161-174 (2017)

40. Pizzorusso, T. et al. Reactivation of ocular dominance plasticity in the adult visual cortex. Science 298, 1248-1251 (2002).

41. Asher, R. A., Morgenstern, D. A., Moon, L. D. \& Fawcett, J. W. Chondroitin sulphate proteoglycans: inhibitory components of the glial scar. Prog. Brain Res. 132, 611-619 (2001).

42. Frischknecht, R., Chang, K. J., Rasband, M. N. \& Seidenbecher, C. I. Neural ECM molecules in axonal and synaptic homeostatic plasticity. Prog. Brain Res. 214 81-100 (2014).

43. Begum, M. R. \& Sng, J. C. G. Molecular mechanisms of experience-dependent maturation in cortical GABAergic inhibition. J. Neurochem. 142, 649-661 (2017)

44. Hou, X. et al. Chondroitin sulfate is required for onset and offset of critical period plasticity in visual cortex. Sci. Rep. 7, 12646 (2017).

45. Lensjo, K. K., Lepperod, M. E., Dick, G., Hafting, T. \& Fyhn, M. Removal of perineuronal nets unlocks juvenile plasticity through network mechanisms of decreased inhibition and increased gamma activity. J. Neurosci. 37 1269-1283 (2017).

46. Stephany, C. E., Ikrar, T., Nguyen, C., Xu, X. \& McGee, A. W. Nogo receptor 1 confines a disinhibitory microcircuit to the critical period in visual cortex. J. Neurosci. 36, 11006-11012 (2016).

47. Varbanov, H. \& Dityatev, A. Regulation of extrasynaptic signaling by polysialylated NCAM: impact for synaptic plasticity and cognitive functions. Mol. Cell. Neurosci. 81, 12-21 (2017).

48. Di Cristo, G. et al. Activity-dependent PSA expression regulates inhibitory maturation and onset of critical period plasticity. Nat. Neurosci. 10, 1569-1577 (2007).

49. Mataga, N., Nagai, N. \& Hensch, T. K. Permissive proteolytic activity for visual cortical plasticity. Proc. Natl. Acad. Sci. USA 99, 7717-7721 (2002).

50. Taha, S. \& Stryker, M. P. Rapid ocular dominance plasticity requires cortical but not geniculate protein synthesis. Neuron 34, 425-436 (2002).

51. Carulli, D. et al. Animals lacking link protein have attenuated perineuronal nets and persistent plasticity. Brain 133, 2331-2347 (2010).

52. Murase, S., Lantz, C. L. \& Quinlan, E. M. Light reintroduction after dark exposure reactivates plasticity in adults via perisynaptic activation of MMP-9. Elife 6(6), e27345 (2017). pii.

53. Pham, T. A. et al. A semi-persistent adult ocular dominance plasticity in visual cortex is stabilized by activated CREB. Learn. Mem. 11, 738-747 (2004).

54. Mower, G. D. \& Kaplan, I. V. Immediate early gene expression in the visual cortex of normal and dark reared cats: differences between fos and egr-1. Brain Res. Mol. Brain Res. 105, 157-160 (2002).

55. Mataga, N., Fujishima, S., Condie, B. G. \& Hensch, T. K. Experiencedependent plasticity of mouse visual cortex in the absence of the neuronal activity-dependent marker egr1/zif268. J. Neurosci. 21, 9724-9732 (2001) 
56. Yang, C. B., Zheng, Y. T., Li, G. Y. \& Mower, G. D. Identification of Munc13-3 as a candidate gene for critical-period neuroplasticity in visual cortex. J. Neurosci. 22, 8614-8618 (2002).

57. Huh, G. S. et al. Functional requirement for class I MHC in CNS development and plasticity. Science 290, 2155-2159 (2000).

58. Sakai, A. et al. Genome-wide target analyses of Otx2 homeoprotein in postnatal cortex. Front. Neurosci. 11, 307 (2017).

59. Bear, M. F. \& Singer, W. Modulation of visual cortical plasticity by acetylcholine and noradrenaline. Nature 320, 172-176 (1986).

60. Kasamatsu, T. \& Pettigrew, J. D. Depletion of brain catecholamines: failure of ocular dominance shift after monocular occlusion in kittens. Science 194 206-209 (1976).

61. Gu, Q. \& Singer, W. Involvement of serotonin in developmental plasticity of kitten visual cortex. Eur. J. Neurosci. 7, 1146-1153 (1995).

62. Kirkwood, A., Rozas, C., Kirkwood, J., Perez, F. \& Bear, M. F. Modulation of longterm synaptic depression in visual cortex by acetylcholine and norepinephrine. J. Neurosci. 19, 1599-1609 (1999).

63. Choi, S. Y. et al. Multiple receptors coupled to phospholipase C gate longterm depression in visual cortex. J. Neurosci. 25, 11433-11443 (2005).

64. Maya Vetencourt, J. F. et al. The antidepressant fluoxetine restores plasticity in the adult visual cortex. Science 320, 385-388 (2008).

65. Guirado, R. et al. Effects of PSA removal from NCAM on the critical period plasticity triggered by the antidepressant fluoxetine in the visual cortex. Front. Cell Neurosci. 10, 22 (2016).

66. Morishita, H., Miwa, J. M., Heintz, N. \& Hensch, T. K. Lynx1, a cholinergic brake, limits plasticity in adult visual cortex. Science 330, 1238-1240 (2010).

67. Cai, S. et al. GABAB receptor-dependent bidirectional regulation of critical period ocular dominance plasticity in cats. PLOS ONE 12, e0180162 (2017).

68. Blundon, J. A. et al. Restoring auditory cortex plasticity in adult mice by restricting thalamic adenosine signaling. Science 356, 1352-1356 (2017).

69. Jiang, B. et al. The maturation of GABAergic transmission in visual cortex requires endocannabinoid-mediated LTD of inhibitory inputs during a critical period. Neuron 66, 248-259 (2010).

70. Huang, S., Gu, Y., Quinlan, E. M. \& Kirkwood, A. A refractory period for rejuvenating GABAergic synaptic transmission and ocular dominance plasticity with dark exposure. J. Neurosci. 30, 16636-16642 (2010).

71. Lemtiri-Chlieh, F. \& Levine, E. S. BDNF evokes release of endogenous cannabinoids at layer $2 / 3$ inhibitory synapses in the neocortex. J. Neurophysiol. 104, 1923-1932 (2010).

72. Zhao, L. \& Levine, E. S. BDNF-endocannabinoid interactions at neocortical inhibitory synapses require phospholipase C signaling. J. Neurophysiol. 111 1008-1015 (2014).

73. Yeh, M. L., Selvam, R. \& Levine, E. S. BDNF-induced endocannabinoid release modulates neocortical glutamatergic neurotransmission. Synapse 71, e21962 (2017).

74. Hong, E. J., McCord, A. E. \& Greenberg, M. E. A biological function for the neuronal activity-dependent component of Bdnf transcription in the development of cortical inhibition. Neuron 60, 610-624 (2008).

75. Sakata, K. et al. Critical role of promoter IV-driven BDNF transcription in GABAergic transmission and synaptic plasticity in the prefrontal cortex. Proc. Natl. Acad. Sci. USA 106, 5942-5947 (2009).

76. Holm, M. M. et al. Mature BDNF, but not proBDNF, reduces excitability of fastspiking interneurons in mouse dentate gyrus. J. Neurosci. 29, 12412-12418 (2009).

77. Zheng, $\mathrm{K}$. et al. TrkB signaling in parvalbumin-positive interneurons is critical for gamma-band network synchronization in hippocampus. Proc. Natl. Acad. Sci. USA 108, 17201-17206 (2011).

78. Bernard, C. et al. Graded Ot×2 activities demonstrate dose-sensitive eye and retina phenotypes. Hum. Mol. Genet. 23, 1742-1753 (2014).

79. Beurdeley, M. et al. Otx2 binding to perineuronal nets persistently regulates plasticity in the mature visual cortex. J. Neurosci. 32, 9429-9437 (2012).

80. Bernard, C. \& Prochiantz, A. Otx2-PNN interaction to regulate cortical plasticity. Neural Plast. 2016, 7931693 (2016).

81. Sun, X. \& Lin, Y. Npas4: linking neuronal activity to memory. Trends Neurosci. 39, 264-275 (2016)

82. Yun, J. et al. Neuronal Per Arnt Sim (PAS) domain protein 4 (NPAS4) regulates neurite outgrowth and phosphorylation of synapsin I. J. Biol. Chem. 288, 2655-2664 (2013).
83. Lin, Y. et al. Activity-dependent regulation of inhibitory synapse development by Npas4. Nature 455, 1198-1204 (2008).

84. Jenks, K. R. et al. Arc restores juvenile plasticity in adult mouse visual cortex. Proc. Natl. Acad. Sci. USA 114, 9182-9187 (2017).

85. Putignano, E. et al. Developmental downregulation of histone posttranslational modifications regulates visual cortical plasticity. Neuron 53, 747-759 (2007).

86. Mellios, N. et al. miR-132, an experience-dependent microRNA, is essential for visual cortex plasticity. Nat. Neurosci. 14, 1240-1242 (2011).

87. Tognini, P., Putignano, E., Coatti, A. \& Pizzorusso, T. Experience-dependent expression of miR-132 regulates ocular dominance plasticity. Nat. Neurosci. 14, 1237-1239 (2011).

88. Koh, D. X. \& Sng, J. C. HDAC1 negatively regulates Bdnf and Pvalb required for parvalbumin interneuron maturation in an experience-dependent manner. J. Neurochem. 139, 369-380 (2016).

89. Nott, A., Cho, S., Seo, J. \& Tsai, L. H. HDAC2 expression in parvalbumin interneurons regulates synaptic plasticity in the mouse visual cortex. Neuroepigenetics 1, 34-40 (2015).

90. Schoop, V. M., Gardziella, S. \& Muller, C. M. Critical period-dependent reduction of the permissiveness of cat visual cortex tissue for neuronal adhesion and neurite growth. Eur. J. Neurosci. 9, 1911-1922 (1997).

91. Muller, C. M. \& Best, J. Ocular dominance plasticity in adult cat visual cortex after transplantation of cultured astrocytes. Nature 342, 427-430 (1989).

92. Olsen, M. L. et al. New insights on astrocyte ion channels: critical for homeostasis and neuron-glia signaling. J. Neurosci. 35, 13827-13835 (2015).

93. Papouin, T., Dunphy, J., Tolman, M., Foley, J. C. \& Haydon, P. G. Astrocytic control of synaptic function. Philos. Trans. R. Soc. Lond. B Biol. Sci. 372 20160154 (2017)

94. Allen, N. J. \& Eroglu, C. Cell biology of astrocyte-synapse interactions. Neuron 96, 697-708 (2017)

95. Navarrete, M. SpringerAmpamp; Araque, A. Endocannabinoids potentiate synaptic transmission through stimulation of astrocytes. Neuron $\mathbf{6 8}, \mathbf{1 1 3 - 1 2 6}$ (2010).

96. Sipe, G. O. et al. Microglial P2Y12 is necessary for synaptic plasticity in mouse visual cortex. Nat. Commun. 7, 10905 (2016).

97. Smith, M. R., et al. Integrative analysis of disease signatures shows inflammation disrupts juvenile experience-dependent cortical plasticity. eNeuro $\mathbf{3}$ ENEURO.0240-16.2016 (2016).

98. Engelhardt, M. et al. Leukemia inhibitory factor impairs structural and neurochemical development of rat visual cortex in vivo. Mol. Cell. Neurosci. 79 81-92 (2017).

99. Lee, W. C. \& Nedivi, E. Extended plasticity of visual cortex in dark-reared animals may result from prolonged expression of cpg15-like genes. J. Neurosci. 22, 1807-1815 (2002).

100. Ciucci, F. et al. Insulin-like growth factor 1 (IGF-1) mediates the effects of enriched environment (EE) on visual cortical development. PLOS ONE 2, e475 (2007).

101. McGee, A. W., Yang, Y., Fischer, Q. S., Daw, N. W. \& Strittmatter, S. M Experience-driven plasticity of visual cortex limited by myelin and Nogo receptor. Science 309, 2222-2226 (2005)

102. Frantz, M. G., Kast, R. J., Dorton, H. M., Chapman, K. S. \& McGee, A. W. Nogo receptor 1 limits ocular dominance plasticity but not turnover of axonal boutons in a model of amblyopia. Cereb. Cortex 26, 1975-1985 (2016).

103. Gu, Y. et al. Neuregulin-dependent regulation of fast-spiking interneuron excitability controls the timing of the critical period. J. Neurosci. 36 10285-10295 (2016)

104. Sun, Y. et al. Neuregulin-1/ErbB4 signaling regulates visual cortical plasticity. Neuron 92, 160-173 (2016).

105. Han, K. S., Cooke, S. F. \& Xu, W. Experience-dependent equilibration of AMPAR-mediated synaptic transmission during the critical period. Cell Rep. 18, 892-904 (2017)

106. Kobayashi, Y., Ye, Z. \& Hensch, T. K. Clock genes control cortical critical period timing. Neuron 86, 264-275 (2015).

107. Atwal, J. K. et al. PirB is a functional receptor for myelin inhibitors of axonal regeneration. Science 322, 967-970 (2008).

108. Syken, J., Grandpre, T., Kanold, P. O. \& Shatz, C. J. PirB restricts oculardominance plasticity in visual cortex. Science 313, 1795-1800 (2006). 
109. Bochner, D. N. et al. Blocking PirB up-regulates spines and functional synapses to unlock visual cortical plasticity and facilitate recovery from amblyopia. Sci. Transl. Med. 6, 258ra140 (2014).

110. Nomura, T. et al. Delayed maturation of fast-spiking interneurons is rectified by activation of the TrkB receptor in the mouse model of fragile $X$ syndrome. J. Neurosci. 37, 11298-11310 (2017).

111. He, L. J. et al. Conditional deletion of Mecp2 in parvalbumin-expressing GABAergic cells results in the absence of critical period plasticity. Nat. Commun. 5, 5036 (2014).

112. Pizzo, R. et al. Lack of Cdkl5 disrupts the organization of excitatory and inhibitory synapses and parvalbumin interneurons in the primary visual cortex. Front. Cell Neurosci. 10, 261 (2016).

113. Aceti, M. et al. Syngap1 haploinsufficiency damages a postnatal critical period of pyramidal cell structural maturation linked to cortical circuit assembly. Biol. Psychiatry 77, 805-815 (2015).

114. Carcea, I. et al. Maturation of cortical circuits requires Semaphorin 7A. Proc. Natl. Acad. Sci. USA 111, 13978-13983 (2014)

115. Allegra, M. et al. Altered GABAergic markers, increased binocularity and reduced plasticity in the visual cortex of Engrailed-2 knockout mice. Front. Cell Neurosci. 8, 163 (2014)

116. Crocker-Buque, A. et al. Altered thalamocortical development in the SAP102 knockout model of intellectual disability. Hum. Mol. Genet. 25, 4052-4061 (2016).

117. Yamamuro, K. et al. Social isolation during the critical period reduces synaptic and intrinsic excitability of a subtype of pyramidal cell in mouse prefrontal cortex. Cereb. Cortex 28, 998-1010 (2018).

118. Li, J. T. et al. Repeated blockade of NMDA receptors during adolescence impairs reversal learning and disrupts GABAergic interneurons in rat medial prefrontal cortex. Front. Mol. Neurosci. 9, 17 (2016).

119. Guirado, R., Perez-Rando, M., Sanchez-Matarredona, D., Castren, E. \& Nacher, J. Chronic fluoxetine treatment alters the structure, connectivity and plasticity of cortical interneurons. Int. J. Neu ropsychopharmacol. 17, 1635-1646 (2014).

120. Sale, A. \& Berardi, N. Active training for amblyopia in adult rodents. Front Behav. Neurosci. 9, 281 (2015).

121. Kalogeraki, E., Greifzu, F., Haack, F. \& Lowel, S. Voluntary physical exercise promotes ocular dominance plasticity in adult mouse primary visual cortex. J. Neurosci. 34, 15476-15481 (2014).

122. Levine, J. N., Chen, H., Gu, Y. \& Cang, J. Environmental enrichment rescues binocular matching of orientation preference in the mouse visual cortex. $J$. Neurosci. 37, 5822-5833 (2017).

123. Baroncelli, L. et al. Experience affects critical period plasticity in the visual cortex through an epigenetic regulation of histone post-translational modifications. J. Neurosci. 36, 3430-3440 (2016).

124. Levi, D. M., Knill, D. C. \& Bavelier, D. Stereopsis and amblyopia: a mini-review. Vision Res. 114, 17-30 (2015).

125. Castano-Castano, S. et al. Transcranial direct current stimulation improves visual acuity in amblyopic Long-Evans rats. Brain Res. 1657, 340-346 (2017).

126. Campana, G., Camilleri, R., Pavan, A., Veronese, A. \& Lo Giudice, G. Improving visual functions in adult amblyopia with combined perceptual training and transcranial random noise stimulation (tRNS): a pilot study. Front. Psychol. 5, 1402 (2014).

127. Mix, A., Hoppenrath, K. \& Funke, K. Reduction in cortical parvalbumin expression due to intermittent theta-burst stimulation correlates with maturation of the perineuronal nets in young rats. Dev. Neurobiol. 75, 1-11 (2015).

128. Rudy, B., Fishell, G., Lee, S., \& Hjerling-Leffler, J. Three groups of interneurons account for nearly $100 \%$ of neocortical GABAergic neurons. Dev. Neurobiol. 71, 45-61 (2011).

129. Sugiyama, S. et al. Experience-dependent transfer of Otx2 homeoprotein into the visual cortex activates postnatal plasticity. Cell 134, 508-520 (2008). 